\title{
Spatio-temporal distribution of soil-transmitted helminth infections in Brazil
}

\author{
Frédérique Chammartin ${ }^{1,2}$, Luiz H Guimarães ${ }^{3}$, Ronaldo GC Scholte ${ }^{4}$, Mara E Bavia ${ }^{5}$, Jürg Utzinger ${ }^{1,2}$ \\ and Penelope Vounatsou ${ }^{1,2^{*}}$
}

\begin{abstract}
Background: In Brazil, preventive chemotherapy targeting soil-transmitted helminthiasis is being scaled-up. Hence, spatially explicit estimates of infection risks providing information about the current situation are needed to guide interventions. Available high-resolution national model-based estimates either rely on analyses of data restricted to a given period of time, or on historical data collected over a longer period. While efforts have been made to take into account the spatial structure of the data in the modelling approach, little emphasis has been placed on the temporal dimension.
\end{abstract}

Methods: We extracted georeferenced survey data on the prevalence of infection with soil-transmitted helminths (i.e. Ascaris lumbricoides, hookworm and Trichuris trichiura) in Brazil from the Global Neglected Tropical Diseases (GNTD) database. Selection of the most important predictors of infection risk was carried out using a Bayesian geostatistical approach and temporal models that address non-linearity and correlation of the explanatory variables. The spatial process was estimated through a predictive process approximation. Spatio-temporal models were built on the selected predictors with integrated nested Laplace approximation using stochastic partial differential equations.

Results: Our models revealed that, over the past 20 years, the risk of soil-transmitted helminth infection has decreased in Brazil, mainly because of the reduction of A. lumbricoides and hookworm infections. From 2010 onwards, we estimate that the infection prevalences with A. lumbricoides, hookworm and T. trichiura are 3.6\%, 1.7\% and 1.4\%, respectively. We also provide a map highlighting municipalities in need of preventive chemotherapy, based on a predicted soil-transmitted helminth infection risk in excess of $20 \%$. The need for treatments in the school-aged population at the municipality level was estimated at 1.8 million doses of anthelminthic tablets per year.

Conclusions: The analysis of the spatio-temporal aspect of the risk of infection with soil-transmitted helminths contributes to a better understanding of the evolution of risk over time. Risk estimates provide the soil-transmitted helminthiasis control programme in Brazil with useful benchmark information for prioritising and improving spatial and temporal targeting of interventions.

Keywords: Soil-transmitted helminth, Ascaris lumbricoides, Hookworm, Trichuris trichiura, Predictive risk mapping, Bayesian geostatistics, Spatio-temporal model, Brazil

\footnotetext{
* Correspondence: penelope.vounatsou@unibas.ch
${ }^{1}$ Department of Epidemiology and Public Health, Swiss Tropical and Public

* Correspondence: penelope.vounatsou@unibas.ch
${ }^{1}$ Department of Epidemiology and Public Health, Swiss Tropical and Public

Health Institute, P.O. Box, CH-4002 Basel, Switzerland

${ }^{2}$ University of Basel, P.O. Box, CH-4003 Basel, Switzerland

Full list of author information is available at the end of the article
}

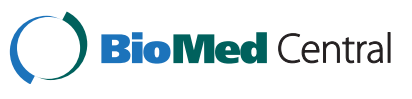

(c) 2014 Chammartin et al.; licensee BioMed Central Ltd. This is an Open Access article distributed under the terms of the Creative Commons Attribution License (http://creativecommons.org/licenses/by/4.0), which permits unrestricted use, distribution, and reproduction in any medium, provided the original work is properly credited. The Creative Commons Public Domain Dedication waiver (http://creativecommons.org/publicdomain/zero/1.0/) applies to the data made available in this article, unless otherwise stated. 


\section{Background}

The nematode worms Ascaris lumbricoides, Trichuris trichiura and the two hookworm species Ancylostoma duodenale and Necator americanus are commonly referred to as soil-transmitted helminths [1]. These nematodes parasitise the human intestine and might lead to chronic infections with clinical consequences that undermine health of affected populations [1-3]. The World Health Organization (WHO) advocates a global control strategy against major helminthiases, emphasising preventive chemotherapy targeting high-risk communities, in combination with health education and sanitation improvement whenever resources allow [4].

Soil-transmitted helminthiases are of considerable public health concern in tropical and subtropical countries, where climatic conditions and poverty-related behaviours favour their transmission [5,6]. South America is not spared $[7,8]$. In Brazil, deworming campaigns were carried out covering up to $60 \%$ of the population but interventions have been interrupted in 2005 [4,9], partially because of the decentralization of the programme [10]. Currently, WHO estimates that 9 million school-aged children in Brazil require preventive chemotherapy and anthelminthic administration of albendazole has been re-implemented in 2013 [4].

Spatial targeting of the population requiring preventive chemotherapy and other interventions is essential to implement tailored and cost-effective control measures. Bayesian geostatistical models are used to establish a statistical relationship between observed prevalence and environmental and socioeconomic risk factors, and predict the risk at unobserved locations, while accounting for spatial heterogeneity through spatially structured random effects [11]. These models have been widely applied to model soil-transmitted helminth risk at different scales [7,12-14]. They are highly parameterised, and therefore estimation of model parameters relies on Markov chain Monte Carlo (MCMC) sampling methods. However, inference requires multiple inversions of the spatially structured variance-covariance matrix and MCMC methods are known to be computationally intensive. Thus, for large datasets, spatial process estimation can rely on low-rank approximation, such as the predictive process $[7,15]$.

By incorporating a temporal trend into the model, changes of the disease risk and pattern over time can be studied $[7,14,16]$. A temporal trend assumes that the infection risk changes over time by a certain amount, which is constant across space. However, the underlying latent spatial process might also vary over time. Bayesian formulations introduced by Knorr-Held [17] allow accounting for space-time interaction with an effect that is spatio-temporally structured through its precision matrix. Hence, such spatio-temporal models are able to estimate the spatial variation with time. The spatio-temporal aspect of helminthiases risk is an under-explored issue, mainly because of computational challenges in estimating highly parameterised models with MCMC algorithms. However, recent developments in Bayesian inference with integrated nested Laplace approximation (INLA) [18] using stochastic partial differential equations (SPDEs) [19] offer new opportunities for accurate fit of complex models at reasonable computational cost and time [20].

Here, we present an analysis of the spatio-temporal distribution of soil-transmitted helminth infection risks in Brazil. Our research extends a recent study that focused on the spatial distribution of soil-transmitted helminth infections in Brazil that was based on a relatively small database covering the period 2005-2009 [8]. We extended the survey period that now spans two decades (1995-2013) and focused on the space-time interactions of the disease patterns. We provide high-resolution spatial estimates of helminth species-specific infection risks and assess annualised deworming needs for school-aged children for Brazil. Historical data was extracted from the Global Neglected Tropical Diseases (GNTD) database [21], and Bayesian spatio-temporal models were fitted in a SPDEs/INLA framework. Predictors included in each model were selected within a Bayesian geostatistical variable selection approach that is well suited for large datasets.

\section{Methods \\ Disease data}

Prevalence survey data pertaining to A. lumbricoides, hookworm and $T$. trichiura in Brazil were extracted from the GNTD database (www.gntd.org). The GNTD database is an open-access platform gathering spatially explicit survey data on soil-transmitted helminthiasis and other neglected tropical diseases identified through systematic searches of readily available electronic databases and grey literature [21,22]. The literature search for relevant soil-transmitted helminth prevalence data in Brazil was updated on 27th November 2013 and includes surveys conducted from 1995 onwards. The reader is referred to previous publications for further details on search strategy, geolocation and data quality appraisal $[7,21]$.

\section{Environmental, socioeconomic and population data}

Table 1 summarises the sources and the spatial and temporal resolutions of environmental, socioeconomic and population data considered in our analysis. A total of 29 variables were taken into account as potential risk factors for soil-transmitted helminth infection. Environmental data included altitude, soil acidity, soil moisture and 19 bioclimatic variables related to temperature and precipitation. Socioeconomic proxies were: human development 
Table 1 Data sources and properties of the predictors explored to model soil-transmitted helminth infection risk in Brazil

\begin{tabular}{|c|c|c|c|}
\hline Data type & Source & Temporal resolution & Spatial resolution \\
\hline Temperature and precipitation $^{a}$ & WorldClimb & $1950-2000$ & $1 \mathrm{~km}$ \\
\hline Altitude & SRTM ${ }^{c}$ & 2000 & $1 \mathrm{~km}$ \\
\hline Soil acidity/soil moisture & ISRIC-WISE ${ }^{d}$ & $1960-2000$ & $10 \mathrm{~km}$ \\
\hline Human influence index (HII) & LTW $^{\mathrm{e}}$ & 2005 & $1 \mathrm{~km}$ \\
\hline Human development index (HDI) & Atlas Brasil $2013^{\mathrm{f}}$ & 2000 and 2010 & Municipality \\
\hline Poor households & Atlas Brasil $2013^{f}$ & 2000 and 2010 & Municipality \\
\hline Rurality, improved water supply, sewage system and waste treatment & Ministério da Saúde ${ }^{g}$ & 2010 & Municipality \\
\hline Population density & GPWFE $^{h}$ & 2010 & $10 \mathrm{~km}$ \\
\hline
\end{tabular}

${ }^{\mathrm{a}} \mathrm{A}$ total of 19 climatic variables related to various factors were considered.

borldClim Global Climate database version 1.4; available at: http://www.worldclim.org/ (accessed: March 2012).

'Shuttle Radar Topography Mission (SRTM); available at: http://www.worldclim.org/ (accessed: March 2012).

dGlobal soil profile data ISRIC-WISE database version 1.2; available at: http://www.isric.org/ (accessed: December 2012).

e Last of the Wild Data version 2, 2005 (LTW-2): Global Human Footprint Dataset (Geographic). Wildlife Conservation (WCS) and Center for International Earth

Science Information Network (CIESIN); available at: http://www.ciesin.org/wildareas/ (accessed: December 2013).

${ }^{f}$ Atlas do Desenvolvimento Humano no Brasil 2013; available at: http://atlasbrasil.org.br/ (accessed: December 2013).

${ }^{9}$ Ministério da Saúde, Brazil; available at: http://www2.datasus.gov.br/DATASUS/index.php (accessed: December 2013).

${ }^{h}$ Gridded population of the world: future estimates (GPWFE): Center for International Earth Science Information Network (CIESIN), UN Food and Agriculture

Organization (FAO) and Centro Internacional de Agricultura Tropical (CIAT); available at: http://sedac.ciesin.columbia.edu/gpw (accessed: December, 2012).

index (HDI), which is a measure of socioeconomic development based on life expectancy, education and income; human influence index (HII), which quantifies human influence on ecosystems; a poverty measure reflected by the percentage of people living with a household monthly income lower than US\$ 60 (poor households); and a measure of rurality expressed by the percentage of rural households within municipalities. In addition, using census data we compiled the proportion of individuals within municipalities with access to improved water supply, sewage system and waste treatment. These last three variables were classified as improved according to the following criteria: (i) sewage system connected to a network or to a septic tank; (ii) water supply from a well or through the network; and (iii) waste collection by a cleaning service.

Survey data were linked to potential risk factors based on their spatial proximity when they were available at fine spatial scale or according to their belonging to municipalities in case they were available at this resolution. Moreover, HDI and percentage of poor household data obtained in 2010 were assigned to prevalence data observed from 2005 onwards, while information obtained in 2000 was related to prevalence data prior to 2005 .

\section{Statistical analysis}

Soil-transmitted helminth infection prevalence data were modelled via binomial logistic regression with spatio-temporal random effects accounting for a latent spatial process varying with time. Exploratory analyses were carried out to assess correlations between potential predictors, as well as to explore their association with observed infection risks. Highly correlated potential risk factors (Pearson's correlation coefficient $>0.9$ ) were grouped, with the aim to include not more than one of them in the models. Continuous predictors were standardised (by substracting their mean and dividing with standard deviation) to obtain estimates of the effects, which are comparable across the predictors.

Details on spatio-temporal model formulation and variable selection are given in the Additional file 1. In brief, risk factors included in the spatio-temporal models were selected through a Bayesian stochastic search variable selection approach [23]. We followed our previous procedure, which consists of selecting within a geostatistical framework the best predictors among highly correlated ones, while addressing nonlinearity of the predictors [16]. We further extended this formulation in applications to large datasets, by estimating the spatial process through a predictive process approximation [15]. The inclusion of a variable in the model was defined as the product of two indicators: the first was assumed to be Bernoulli distributed and suggests the inclusion of the group of highly correlated variables, whilst the second followed a categorical prior distribution for selecting a single predictor within the group. In addition, regression coefficients were a priori parameterised with parameter expanded normal mixture of inverse-gamma (peNMIG) distributions [24], which ensure a rigorous selection of categorical variables. Models with the highest posterior probability identified the predictors to include in the final models. 
Spatio-temporal distribution of soil-transmitted helminth infection risk was modelled using the methodology developed by Cameletti et al. [25] for spatio-temporal modelling. SPDEs were used to represent a Matérn spatiotemporal Gaussian field (GF) as a Gaussian Markov random field (GMRF), which in turn allowed an INLA algorithm to estimate model parameters. This approach provides considerable advantages in terms of computational cost compared to traditional MCMC algorithms. The spatio-temporal GF is characterised by a first-order autoregressive temporal effect and another temporally independent effect assumed to arise from a zero mean multivariate normal distribution with spatio-temporal covariance function of the Matérn family for identical time periods.

We further predict the risk of infection with individual soil-transmitted helminth species over a grid of 381,881 pixels $(5 \times 5 \mathrm{~km}$ spatial resolution). To validate our models, we re-fitted our spatiotemporal models on a randomly selected subset of approximately $80 \%$ of the data, and compared model-based estimated risks with the remaining $20 \%$ observed prevalences. Model predictive ability was measured by the proportion of correctly predicted values within the $k$ th highest posterior density (HPD) interval with $k \%$ probability coverage of the posterior distribution varying from $50 \%$ to $95 \%$. We used the mean error (ME) to assess the prediction bias.

\section{Population-adjusted risk and estimated treatment needs for school-aged children}

The overall risk of soil-transmitted helminth infection was calculated for each of the samples of the predictive distribution, at each pixel, with a simple probabilistic model of combined infection divided by a factor of 1.06 [26]. To calculate populationadjusted risks, we multiplied predicted risks by the population at pixel level, summed them up over areas of interest, and divided them by the population of those areas.

Annualised treatment needs for school-aged children (age range: 5-14 years) for preventive chemotherapy were estimated by considering one treatment per year for children living in low-risk municipalities (population-adjusted risk between 20 and 50\%) and two treatments for children living in high-risk areas (population-adjusted risk $\geq 50 \%$ ), following WHO guidelines [27]. The schoolaged population was estimated to represent $16.9 \%$ of the total population in Brazil, according to 2010 census data (http://www.ibge.gov.br/).

\section{Ethics statement}

All data were obtained from existing databases without personal identifiers. Here, the data were further analysed to deepen our understanding of the spatio-temporal distribution of soil-transmitted helminth infections in Brazil. Hence, there were no specific ethical considerations for the current analysis.

\section{Results}

From 1995 onwards, we obtained spatially explicit information about prevalence of A. lumbricoides, T. trichiura and hookworm across Brazil for 10,513, 10,497 and 10,492 locations, respectively. The frequency distribution of individual soil-transmitted helminth species surveys, stratified by year, is depicted in Figure 1. The datasets included 1,587, 1,572 and 1,570 unique locations for A. lumbricoides, T. trichiura and hookworm, respectively. Data were aggregated over four time periods, i.e. (i) 1995-1999; (ii) 2000-2004; (iii) 2005-2009; and (iv) from 2010 onwards. Figure 2 shows the spatial distribution of the observed prevalence, stratified by soil-transmitted helminth species and time periods. As illustrated in Figure 3, a reduction of the overall raw prevalence was observed over the four periods, with the
(A)

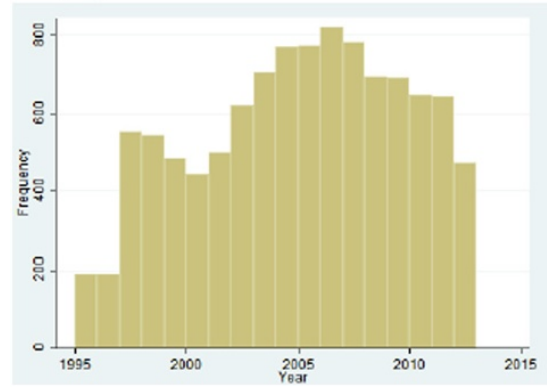

(B)

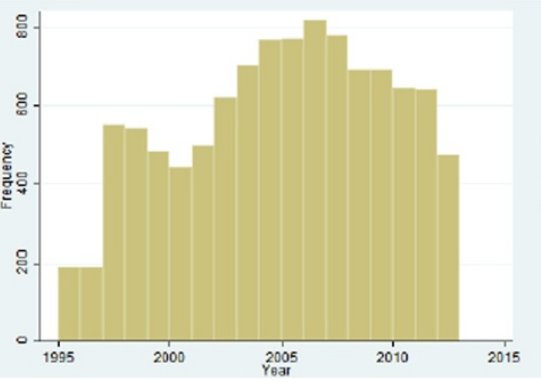

(C)

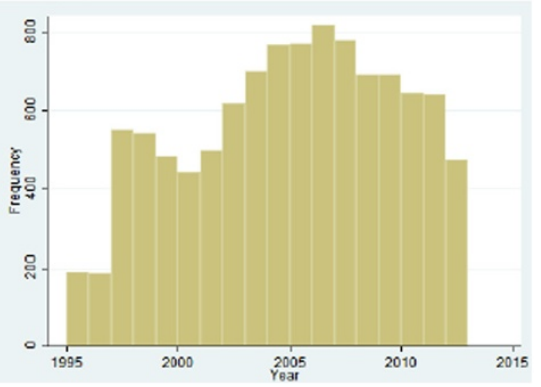

Figure 1 Frequency distribution of soil-transmitted helminth survey data in Brazil from 1995 to 2013, stratified by year. (A) A. lumbricoides, (B) T. trichiura and (C) hookworm. 


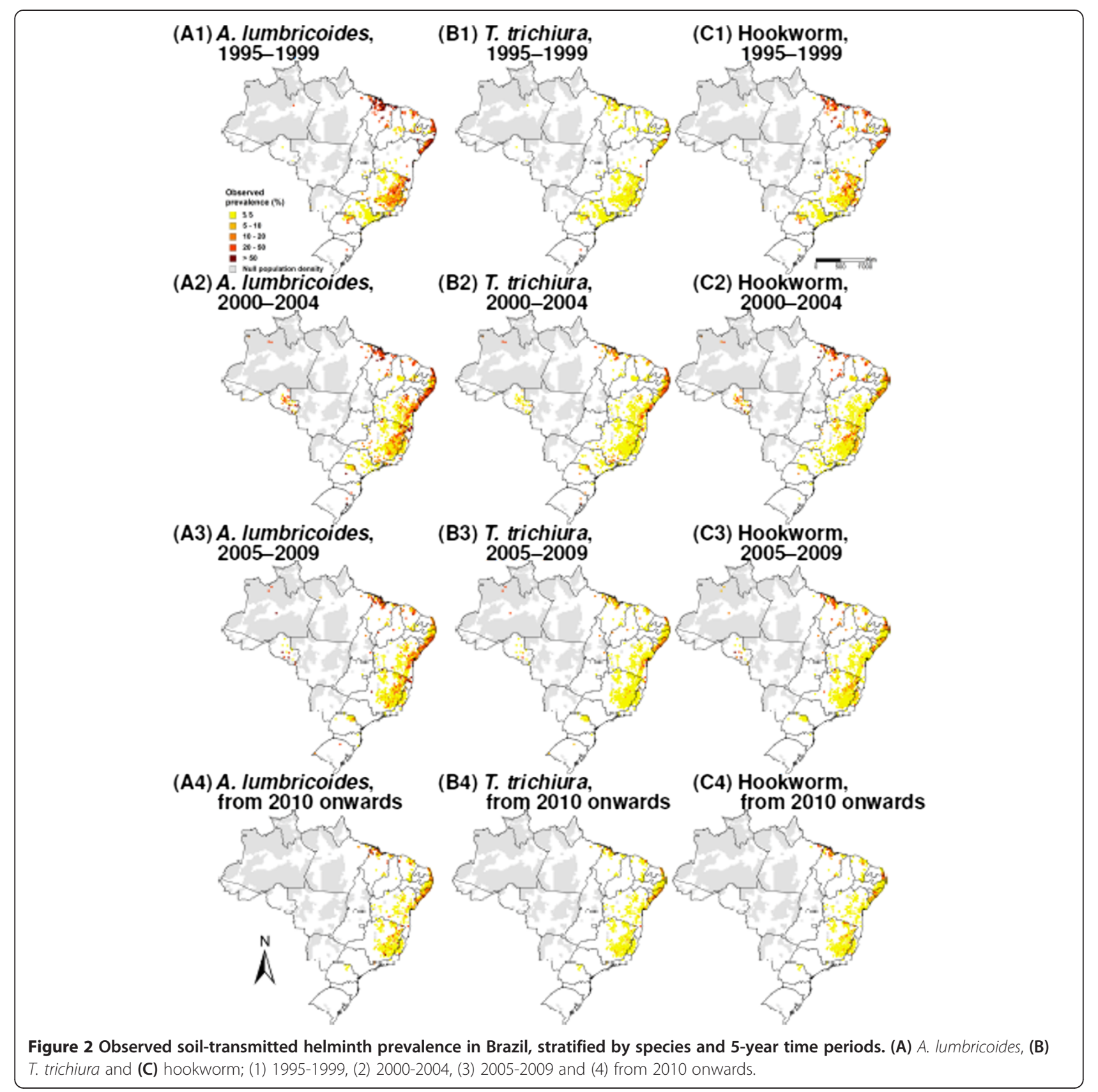

exception of $T$. trichiura infection, which showed peak prevalence in 2000-2004.

Results of the variable selection are given in Table 2. Out of the 29 potential predictors investigated, our variable selection procedure identified 14, 13 and 12 variables as being important for T. trichiura, A. lumbricoides and hookworm, respectively with model posterior probabilities of $93.5 \%, 44.8 \%$ and $25.3 \%$. The selected variables were subsequently used to build spatio-temporal models.
Parameter estimates of spatio-temporal multiple regression models, together with the ones of bivariate logistic associations with standard error clustered at location-level are presented for each soil-transmitted helminth species in Tables 3, 4 and 5. Results of bivariate logistic regressions show associations of the selected predictors with observed risk. Temperature and precipitation usually favour the risk of soil-transmitted helminthiasis, as reflected by the positive bivariate associations of temperature during warmest and coldest 


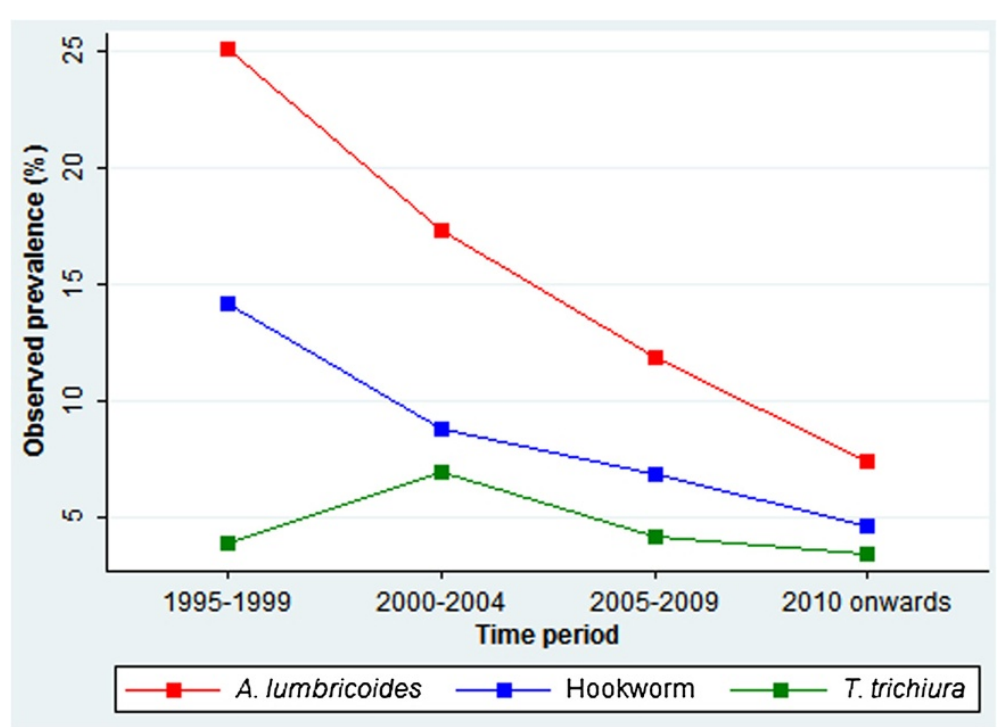

Figure 3 Temporal trend and observed national prevalences for A. lumbricoides, T. trichiura and hookworm infections in Brazil.

quarters and precipitation in coldest quarter and coldest month. However, precipitation during the warmest quarter was negatively associated with the risk of infection with any of the three soil-transmitted helminth species. Furthermore, important temperature and precipitation oscillations show a negative association with soil-transmitted helminth infection odds, as suggested by the effects of diurnal and yearly temperature ranges, low isothermality, as well as temperature and precipitation seasonality. The three infection risks were positively associated to proxies of poverty, as reflected by the positive effect of the percentage of poor households and the negative association of HDI.

In the spatio-temporal model, the odds of A. lumbricoides infection risk was positively associated with yearly precipitation, precipitation in driest quarter, soil $\mathrm{pH}$ (5.35-5.65), poor households and HDI, and negatively associated with mean diurnal temperature range, precipitation in warmest quarter and coldest quarter $(80-300 \mathrm{~mm})$.

For T. trichiura, the predictors with an important positive effect on the odds of the risk were: isothermality, precipitation in driest month, wettest, warmest and coldest quarters, soil pH (5.35-5.65), HDI, HII and poor households. On the other hand, the odds of T. trichiura infection were negatively associated with yearly temperature range, precipitation seasonality, soil moisture $(\geq 80 \%)$ and rural households.

Hookworm infection odds increased with average temperature of warmest month, temperature seasonality, as well as with precipitation in the wettest quarter. On the other hand, precipitation in the warmest and driest quarter, precipitation seasonality, soil moisture, $\mathrm{pH}$ and HDI were negatively associated with the risk of hookworm infection.

The estimates of the effects of the survey periods indicate a decreasing trend for both A. lumbricoides and hookworm infection risks in the period studied, i.e. from 1995 onwards until late 2013. For T. trichiura, there was no important effect of survey period after 2010 compared to the preceding decade.

Figure 4 shows the results of model validation. The risks of soil-transmitted helminth infection were correctly predicted within a $95 \%$ credible interval for $77 \%$ of the tested data for A. lumbricoides, $70 \%$ for T. trichiura and $69 \%$ for hookworm. The ME was -3.03\%, $-2.26 \%$ and $-2.75 \%$ for the three species, respectively, suggesting that our models slightly underestimate the observed prevalences.

Model-based predictions of the geographical distribution of the three soil-transmitted helminth species considered in our analyses are presented in Figure 5, for each of the four time periods. From 2010 onwards, A. lumbricoides infection presents larger risk areas compared to the other two species, with higher risk in the northern part of the country. The highest risk for T. trichiura was found in the north-western part of Brazil, while the risk for hookworm was higher along the northern coast. Our maps also highlight the temporal evolution of the risk for an infection with any of these three soil-transmitted helminth species over the past 20 years. Apparent shrinkage of high-risk areas was observed for $A$. 
Table 2 Variables selected by a Bayesian variable selection approach applied within the geostatistical logistic regression model

$\begin{array}{lll}\text { A. lumbricoides infection } & \text { T. trichiura infection } & \text { Hookworm infection }\end{array}$

\section{Group 1}

Yearly mean temperature ${ }^{a}$

0

Maximum temperature of warmest month ${ }^{\mathrm{a}}$

Minimum temperature of coldest month ${ }^{\mathrm{a}}$

Mean temperature of wettest quarter

Mean temperature of driest quarter

Mean temperature of warmest quarter ${ }^{a}$

Mean temperature of coldest quarter ${ }^{a}$

\section{Group 2}

Mean diurnal temperature range ${ }^{b}$

Yearly temperature range $e^{a, b}$

\section{Group 3}

Isothermality

Temperature seasonality

\section{Group 4}

Yearly precipitation ${ }^{a}$

Precipitation in wettest month

Precipitation in wettest quarter ${ }^{\mathrm{a}}$

\section{Group 5}

Precipitation in driest month ${ }^{\mathrm{a}, \mathrm{c}}$

Precipitation in driest quarter ${ }^{c}$

\section{Moderately correlated}

Precipitation seasonality

Precipitation in warmest quarter ${ }^{b}$

Precipitation in coldest quarter ${ }^{\mathrm{b}, \mathrm{c}}$

Altitude

Soil moisture $\mathrm{e}^{\mathrm{a}, \mathrm{b}, \mathrm{c}}$

Soil $\mathrm{pH}^{\mathrm{b}, \mathrm{c}}$

Human development index (HDI)

Human influence index ${ }^{\mathrm{b}}$ (HII)

Rural households $s^{\mathrm{b}, \mathrm{c}}$

Improved sanitation

Improved water supply $\mathrm{a}, \mathrm{b}, \mathrm{c}$

Improved waste collection ${ }^{\text {b }}$

Poor households

Survey period

Posterior probability (\%)

${ }^{\mathrm{a}}$ Categorised for $T$. trichiura.

${ }^{\mathrm{b}}$ Categorised for hookworm.

'Categorised for A. lumbricoides.

$x$ (selected), 0 (not selected).

The best model selected by the geostatistical variable selections is presented for each soil-transmitted helminth species, together with its posterior probability.

lumbricoides and hookworm. Spatial correlation was estimated around $30 \mathrm{~km}$ for each of the three soiltransmitted helminth species, and spatial variance extended from 5.07 to 9.68. Temporal autocorrelation was generally weak, suggesting that temporal structure was explained by the temporal trend, as well as 
Table 3 Parameter estimates of bivariate and Bayesian spatio-temporal logistic models for A. lumbricoides infection risk in Brazil

\begin{tabular}{|c|c|c|}
\hline A. lumbricoides infection & $\begin{array}{l}\text { Bivariate logistic }{ }^{\dagger} \\
\text { OR }(95 \% \mathrm{Cl})\end{array}$ & $\begin{array}{c}\text { Spatio-temporal model } \\
\text { OR }(95 \% \mathrm{BCl})\end{array}$ \\
\hline \multicolumn{3}{|l|}{ Survey period } \\
\hline 1995-1999 & 1.00 & 1.00 \\
\hline 2000-2004 & $0.62(0.56 ; 0.69)^{*}$ & $0.60(0.51 ; 0.70)^{*}$ \\
\hline $2005-2009$ & $0.40(0.34 ; 0.47)^{*}$ & $0.34(0.28 ; 0.40)^{*}$ \\
\hline From 2010 onwards & $0.24(0.19 ; 0.30)^{*}$ & $0.14(0.12 ; 0.17)^{*}$ \\
\hline Mean temperature of warmest quarter & $1.91(1.73 ; 2.12)^{*}$ & $0.98(0.77 ; 1.24)$ \\
\hline Mean diurnal temperature range & $0.55(0.50 ; 0.61)^{*}$ & $0.83(0.73 ; 0.95)^{*}$ \\
\hline Isothermality & $1.39(1.25 ; 1.55)^{*}$ & $1.01(0.90 ; 1.13)$ \\
\hline Yearly precipitation & $1.44(1.32 ; 1.57)^{*}$ & $1.62(1.43 ; 1.83)^{*}$ \\
\hline \multicolumn{3}{|l|}{ Precipitation in driest quarter ( $\mathrm{mm}$ ) } \\
\hline$<50$ & 1.00 & 1.00 \\
\hline $50-95$ & $1.04(0.75 ; 1.46)$ & $1.56(1.27 ; 1.92)^{*}$ \\
\hline$\geq 95$ & $1.83(1.34 ; 2.51)^{*}$ & $1.36(1.01 ; 1.84)^{*}$ \\
\hline Precipitation seasonality & $0.88(0.79 ; 0.97)^{*}$ & $0.92(0.82 ; 1.02)$ \\
\hline Precipitation in warmest quarter & $0.53(0.47 ; 0.59)^{*}$ & $0.65(0.55 ; 0.76)^{*}$ \\
\hline \multicolumn{3}{|l|}{ Precipitation in coldest quarter (mm) } \\
\hline$<80$ & 1.00 & 1.00 \\
\hline $80-300$ & $1.32(0.98 ; 1.77)$ & $0.65(0.52 ; 0.81)^{*}$ \\
\hline$\geq 300$ & $4.21(3.25 ; 5.45)^{*}$ & $0.92(0.66 ; 1.30)$ \\
\hline Altitude & $0.49(0.44 ; 0.55)^{*}$ & $0.82(0.64 ; 1.06)$ \\
\hline \multicolumn{3}{|l|}{ Soil moisture (\%) } \\
\hline$<50$ & 1.00 & 1.00 \\
\hline $50-80$ & $1.52(1.21 ; 1.91)^{*}$ & $1.16(0.96 ; 1.40)$ \\
\hline$\geq 80$ & $0.92(0.69 ; 1.22)^{*}$ & $1.01(0.77 ; 1.33)$ \\
\hline \multicolumn{3}{|l|}{ Soil pH } \\
\hline$<5.35$ & 1.00 & 1.00 \\
\hline $5.35-5.65$ & $0.70(0.54 ; 0.91)^{*}$ & $1.59(1.36 ; 1.86)^{*}$ \\
\hline$\geq 5.65$ & $0.76(0.59 ; 0.97)^{*}$ & $0.88(0.74 ; 1.06)$ \\
\hline Human development index (HDI) & $0.62(0.56 ; 0.69)^{*}$ & $1.29(1.12 ; 1.49)^{*}$ \\
\hline \multirow[t]{2}{*}{ Poor households } & $1.94(0.68 ; 2.25)$ & $1.81(1.52 ; 2.15)^{*}$ \\
\hline & & Median $(95 \% \mathrm{BCI})$ \\
\hline Temporal autocorrelation & & $0.03(-0.02 ; 0.07)$ \\
\hline Spatial variance & & $5.07(4.73 ; 5.31)$ \\
\hline Spatial range $(\mathbf{k m})$ & & $30.2(28.1 ; 35.2)$ \\
\hline
\end{tabular}

${ }^{\dagger}$ With standard error clustered at location level.

"Significant based on $95 \% \mathrm{Cl}$ or BCl.

OR: odds ratio; $95 \%$ Cl: lower and upper bound of a 95\% confidence interval; 95\% BCl: lower and upper bound of a 95\% Bayesian credible interval.

by changes in HDI and percentage of poor households over time.

Predicted population-adjusted risk estimates in Brazil are given for each survey period analysed (Table 6). Based on predictions from 2010 onwards, we estimated that 10.9 million people were infected with soil- transmitted helminths in Brazil (population-adjusted risk $=6.0 \%$; 95\% Bayesian credible interval $(\mathrm{BCI})$ : 5.4$6.9 \%)$. Single species infection population-adjusted risks were estimated at $3.6 \%$ for A. lumbricoides (95\% BCI: 3.0-4.3\%), $1.7 \%$ for hookworm (95\% BCI: $1.4-2.3 \%$ ), and $1.4 \%$ for $T$. trichiura (95\% BCI: 1.1-1.7\%). Low-risk 
Table 4 Parameter estimates of bivariate and Bayesian spatio-temporal logistic models for T. trichiura infection risk in Brazil

\begin{tabular}{lcc}
\hline T. trichiura infection & Bivariate logistic $^{\dagger}$ & Spatio-temporal model \\
OR $(95 \% \mathrm{CI})$ & $95 \% \mathrm{BCI})$
\end{tabular}
OR $(95 \% \mathrm{Cl})$ OR $(95 \% \mathrm{BCl})$

Survey period

1995-1999

2000-2004

2005-2009

From 2010 onwards

Yearly temperature range $\left({ }^{\circ} \mathrm{C}\right)$

$<13$

$13-18$

$\geq 18$

Mean temperature of coldest quarter $\left({ }^{\circ} \mathrm{C}\right)$

$<19$

$19-22$

$\geq 22$

Isothermality

Precipitation seasonality

Precipitation in wettest quarter ( $\mathrm{mm}$ )

$<560$

$560-680$

$\geq 680$

Precipitation in warmest quarter $(\mathrm{mm})$

$<250$

$250-440$

$\geq 440$

Precipitation in driest month $(\mathrm{mm})$

$<14$

$14-16$

$\geq 16$

Precipitation in coldest quarter $(\mathrm{mm})$

$<80$
$80-300$
$\geq 300$

Soil moisture (\%)

$<50$

$50-80$

$\geq 80$

Soil pH

$<5.35$

5.35-5.65

$\geq 5.65$

Human development index (HDI)

Human influence index (HII)
1.00

$1.83(1.50 ; 2.23)^{*}$

$1.05(0.82 ; 1.36)$

$0.87(0.64 ; 1.17)$

1.00

$0.19(0.15 ; 0.25)^{*}$

$0.12(0.09 ; 0.17)^{*}$

1.00

$3.03(2.25 ; 4.07)^{*}$

$7.97(6.09 ; 10.42)^{*}$

$1.28(1.15 ; 1.42)^{*}$

$0.70(0.64 ; 0.76)^{*}$

1.00

$1.03(0.74 ; 1.45)$

$1.72(1.24 ; 2.40)^{*}$

1.00

$0.62(0.47 ; 0.83)^{*}$

$0.18(0.13 ; 0.24)^{*}$

1.00

$1.07(0.70 ; 1.64)$

$3.21(2.16 ; 4.79)^{*}$

1.00

$2.04(1.38 ; 3.02)^{*}$

$7.67(5.48 ; 10.75)^{*}$

1.00

$1.56(1.15 ; 2.13)^{*}$

$0.91(0.54 ; 1.52)$

1.00

$0.54(0.38 ; 0.77)^{*}$

$0.60(0.45 ; 0.80)^{*}$

$0.74(0.65 ; 0.84)^{*}$

1.00
1.00

$3.47(2.74 ; 4.40)^{*}$

$1.66(1.30 ; 2.10)^{*}$

$0.93(0.71 ; 1.21)$

1.00

$0.34(0.25 ; 0.47)^{*}$

$0.34(0.22 ; 0.53)^{*}$

1.00

$0.83(0.61 ; 1.13)$

$1.42(0.92 ; 2.18)$

$1.27(1.09 ; 1.48)^{*}$

$0.82(0.68 ; 0.98)^{*}$

1.00

$1.65(1.22 ; 2.24)^{*}$

$2.11(1.48 ; 3.00)^{*}$

1.00

$1.50(1.14 ; 1.99)^{*}$

$2.45(1.46 ; 4.11)^{*}$

1.00

$1.48(1.10 ; 1.97)^{*}$

$2.00(1.32 ; 3.05)^{*}$

1.00

$1.13(0.82 ; 1.55)$

$1.80(1.11 ; 2.92)^{*}$

1.00

$0.82(0.61 ; 1.09)$

$0.57(0.38 ; 0.86)^{*}$

1.00

$1.52(1.20 ; 1.92)^{*}$

$0.87(0.67 ; 1.12)$

$1.45(1.17 ; 1.79)^{*}$

1.00 


\begin{tabular}{|c|c|c|}
\hline $20-26$ & $1.39(1.01 ; 1.91)^{*}$ & $1.49(1.20 ; 1.86)^{*}$ \\
\hline$\geq 26$ & $2.14(1.57 ; 2.91)^{*}$ & $1.86(1.46 ; 2.37)^{*}$ \\
\hline \multicolumn{3}{|l|}{ Rural households (\%) } \\
\hline$<25$ & 1.00 & 1.00 \\
\hline $25-50$ & $1.01(0.74 ; 1.39)$ & $0.86(0.68 ; 1.09)$ \\
\hline$\geq 50$ & $0.61(0.44 ; 0.74)^{*}$ & $0.66(0.51 ; 0.85)^{*}$ \\
\hline \multirow[t]{2}{*}{ Poor households } & $1.54(1.27 ; 1.87)^{*}$ & $2.06(1.58 ; 2.68)^{*}$ \\
\hline & & Median $(95 \% \mathrm{BCl})$ \\
\hline Temporal autocorrelation & & $-0.05(-0.10 ; 0.00)$ \\
\hline Spatial variance & & $9.68(9.27 ; 10.03)$ \\
\hline Spatial range (km) & & $32.2(29.9 ; 33.9)$ \\
\hline
\end{tabular}

${ }^{\dagger}$ With standard error clustered at location level.

"Significant based on $95 \% \mathrm{Cl}$ or $\mathrm{BCl}$.

OR: odds ratio; $95 \%$ Cl: lower and upper bound of a 95\% confidence interval; 95\% BCl: lower and upper bound of a 95\% Bayesian credible interval.

(population-adjusted risk 20-50\%) and high-risk (population-adjusted risk $\geq 50 \%$ ) municipalities are highlighted in Figure 6. The highest population-adjusted risk of soiltransmitted helminthiasis was found along the northern coast. We estimated that 1.8 million doses of anthelminthic treatments are required for preventive chemotherapy targeting school-aged children at municipality level in Brazil.

\section{Discussion}

The current study focuses on the spatio-temporal distribution of $A$. lumbricoides, hookworm and T. trichiura risk in Brazil and therefore complements and expands upon a recent study that investigated spatial patterns [8]. We present predictive risk maps at high spatial resolution from 1995 onwards at 5-year increments. Additionally, we provide a map that highlights municipalities that require preventive chemotherapy targeting schoolaged children according to recommendations put forward by WHO [4]. Our analyses provide new insight into spatio-temporal risk profiling of helminthiasis based on a large ensemble of geolocated survey data by considering space-time interactions.

We provide model-based evidence of a decrease of A. lumbricoides and hookworm infection risks over the past 20 years in Brazil. Interestingly, the temporal evolution of the third common soil-transmitted helminth species - T. trichiura - increased after 2000, started to decline from 2005 onwards, and finally reached similar levels to the situation in 1995-1999 from 2010 onwards. We believe that the main reasons explaining the lower risk of A. lumbricoides and hookworm from 2010 onwards compared to the situation 20 years ago are the social and economic development, coupled with deworming activities. Nevertheless, it is important to note that no mass deworming activities were carried out by the Ministry of Health $(\mathrm{MoH})$ in Brazil from 2005 to $2011[4,9]$. The question arises why a similar decline was not observed for T. trichiura. Differences may reflect differential efficacies of the widely used deworming drugs albendazole and mebendazole. While both drugs show high cure and egg reduction rates against $A$. lumbricoides, and albendazole shows satisfactory efficacy against hookworm, neither drug results in high efficacy against T. trichiura $[28,29]$. These differences might explain the delayed risk change profile for T. trichiura.

Our predictive risk maps highlight that high-risk areas of $A$. lumbricoides and T. trichiura infections occur in the north-western part and along the eastern coast of Brazil, while high-risk areas of hookworm infection is concentrated along the northern coast. This is coherent with patterns highlighted by two previous analyses $[7,8]$. However, our population-adjusted estimates for the period 2005-2009 of 7.9\% for A. lumbricoides, 2.5\% for T. trichiura and $2.8 \%$ for hookworm are smaller than those based on predictions from 2005 onwards stemming from a spatial analysis of South America (i.e. 14.3\% for A. lumbricoides, $10.1 \%$ for T. trichiura and $12.3 \%$ for hookworm) [7]. These differences might be explained by the inclusion of socioeconomic factors in the current analysis. Our previous work did not include poverty indicators due to the difficulty of deriving consistent measures among different countries. In comparison to a temporal trend included as a covariate, which indicates the change of magnitude of the risk over time $[7,14]$, spatio-temporal models, as developed in this analysis, highlight the changes in the geographical patterns of risk over time. Hence, our analysis highlights the importance 
Table 5 Parameter estimates of bivariate and Bayesian spatio-temporal logistic models for hookworm infection risk in Brazil

\begin{tabular}{|c|c|c|}
\hline Hookworm infection & $\begin{array}{c}\text { Bivariate logistic }^{\dagger} \\
\text { OR }(95 \% \mathrm{Cl})\end{array}$ & $\begin{array}{c}\text { Spatio-temporal model } \\
\text { OR }(95 \% \mathrm{BCI}) \\
\end{array}$ \\
\hline \multicolumn{3}{|l|}{ Survey period } \\
\hline 1995-1999 & 1.00 & 1.00 \\
\hline 2000-2004 & $0.58(0.50 ; 0.68)^{*}$ & $0.54(0.43 ; 0.68)^{*}$ \\
\hline 2005-2009 & $0.44(0.36 ; 0.54)^{*}$ & $0.28(0.22 ; 0.35)^{*}$ \\
\hline From 2010 onwards & $0.29(0.22 ; 0.39)^{*}$ & $0.13(0.10 ; 0.17)^{*}$ \\
\hline Mean temperature of warmest quarter & $2.00(1.68 ; 2.37)^{*}$ & $1.50(1.10 ; 2.05)^{*}$ \\
\hline Temperature seasonality & $0.54(0.43 ; 0.67)^{*}$ & $1.48(1.21 ; 1.81)^{*}$ \\
\hline \multicolumn{3}{|l|}{ Yearly temperature range $\left({ }^{\circ} \mathrm{C}\right)$} \\
\hline$<13$ & 1.00 & 1.00 \\
\hline $13-18$ & $0.61(0.44 ; 0.86)^{*}$ & $0.98(0.69 ; 1.37)$ \\
\hline$\geq 18$ & $0.20(0.15 ; 0.27)^{*}$ & $0.94(0.60 ; 1.48)$ \\
\hline Precipitation in coldest quarter & $1.62(1.43 ; 1.85)^{*}$ & $0.89(0.69 ; 1.15)$ \\
\hline Precipitation in warmest quarter & $0.44(0.35 ; 0.55)^{*}$ & $0.43(0.33 ; 0.55)^{*}$ \\
\hline Precipitation seasonality & $1.29(1.15 ; 1.45)^{*}$ & $0.61(0.49 ; 0.77)^{*}$ \\
\hline Precipitation in driest quarter & $0.88(0.76 ; 1.01)$ & $0.74(0.62 ; 0.87)^{*}$ \\
\hline Precipitation in wettest quarter & $1.54(1.31 ; 1.80)^{*}$ & $3.59(2.79 ; 4.62)^{*}$ \\
\hline Altitude & $0.56(0.48 ; 0.67)^{*}$ & $0.99(0.71 ; 1.37)$ \\
\hline \multicolumn{3}{|l|}{ Soil moisture (\%) } \\
\hline$<50$ & 1.00 & 1.00 \\
\hline $50-80$ & $0.89(0.64 ; 1.23)$ & $0.52(0.40 ; 0.67)^{*}$ \\
\hline$\geq 80$ & $0.49(0.31 ; 0.78)^{*}$ & $0.30(0.20 ; 0.43)^{*}$ \\
\hline Soil pH & $0.87(0.76 ; 1.00)$ & $0.77(0.70 ; 0.86)^{*}$ \\
\hline \multirow[t]{2}{*}{ Human development index (HDI) } & $0.58(0.53 ; 0.63)^{*}$ & $0.74(0.68 ; 0.82)^{*}$ \\
\hline & & Median $(95 \% \mathrm{BCl})$ \\
\hline Temporal autocorrelation & & $0.01(-0.07 ; 0.06)$ \\
\hline Spatial variance & & $8.92(8.42 ; 9.43)$ \\
\hline Spatial range (km) & & $29.7(28.0 ; 31.4)$ \\
\hline
\end{tabular}

${ }^{\dagger}$ With standard error clustered at location level.

*Significant based on $95 \% \mathrm{Cl}$ or BCl.

OR: odds ratio; $95 \%$ Cl: lower and upper bound of a $95 \%$ confidence interval; $95 \%$ BCl: lower and upper bound of a 95\% Bayesian credible interval.

of considering the temporal aspect of infection risk, especially in a country like Brazil, where socioeconomic conditions have considerably improved and infectious diseases risk has declined over time [7,30]. In comparison to Scholte et al. [8], who analysed a restricted dataset with data provided by the national schistosomiasis control programme for the period 2005-2009, we estimated considerably smaller risks for both $A$. lumbricoides $(7.9 \%$ versus $15.6 \%)$ and $T$. trichiura ( $2.5 \%$ versus $10.1 \%)$. We explain these differences by a considerably higher spatial coverage of our data. Recently, Pullan and colleagues [6] estimated the global risk of soil-transmitted helminth infections for the year 2010 based on empirical approaches, which do not account for small-scale spatial variation. For Brazil, they estimated a risk between 1\% and $10 \%$ for $T$. trichiura and hookworm infections, which is comparable to our estimates of $1.4 \%$ and $1.7 \%$ from 2010 onwards. However, we estimated a risk of $3.6 \%$ for A. lumbricoides, whilst Pullan et al. [6] estimates a risk between $10 \%$ and $20 \%$. This difference highlights the importance of capturing small-scale variation in estimating the risk of helminth infection and other neglected tropical diseases.

Parameter estimates of the spatio-temporal models reflect the climatic suitability and socioeconomic conditions that favour soil-transmitted helminthiasis transmission in Brazil. Each soil-transmitted helminth species risk is influenced by complex interactions of the predictors selected by our variable selection approach. In particular, our 


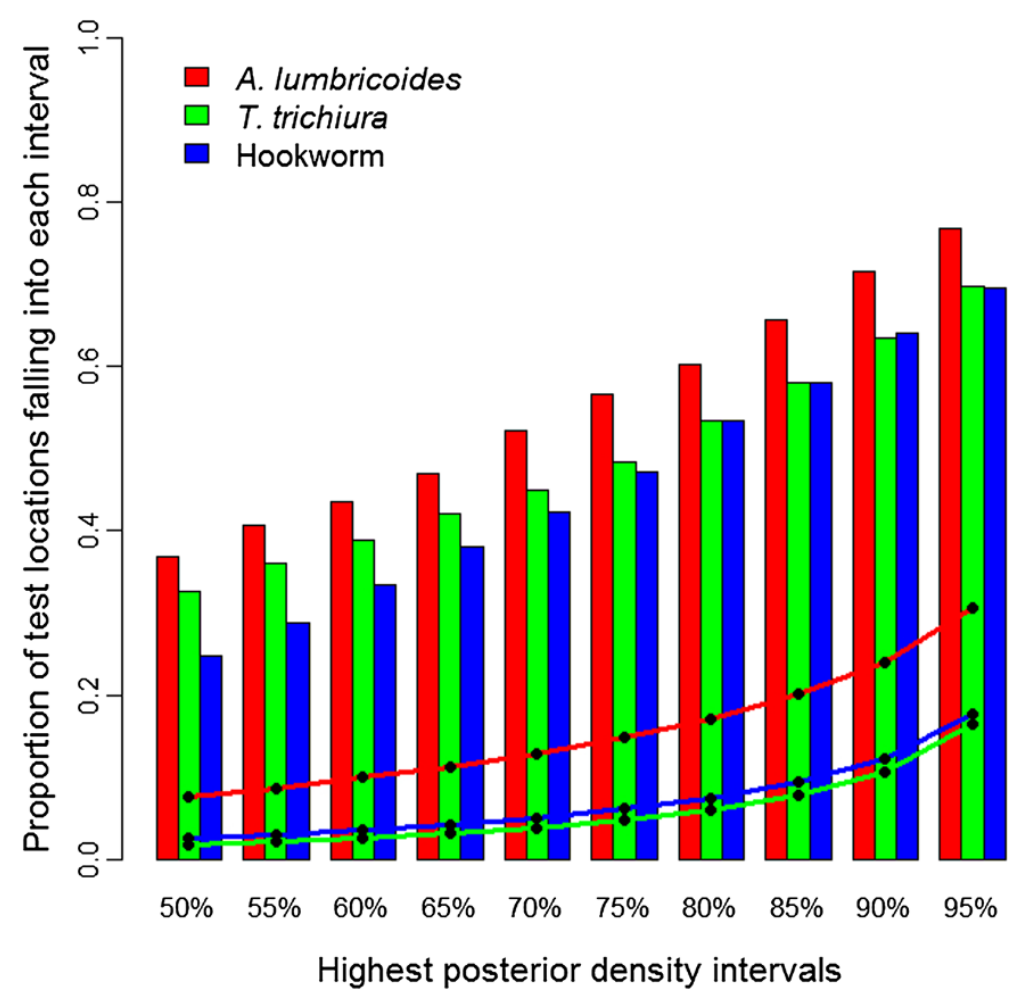

Figure 4 Model validation results. Proportion of surveys with prevalence of infection falling in the predicted highest posterior density (HPD) intervals (bar plots) for A. lumbricoides, T. trichiura and hookworm. The line plots show the corresponding width of the predicted HPD region.

analysis confirms that warm and humid conditions are suitable for soil-transmitted helminth egg and larval development $[31,32]$. Positive associations of precipitation were observed for the three soil-transmitted helminth species and temperature was an important risk factor for hookworm. Extreme weather conditions could adversely affect development and survival of helminthic free-living stages. Indeed, larvae optimally hatch within certain temperature limits [33], suggesting that extreme temperatures might impair their development. Larger range of temperature during the day showed a negative effect in T. trichiura and A. lumbricoides models, while strong isothermality positively impacts the risk of T. trichiura, confirming this hypothesis. Furthermore, it has been speculated that heavy rainfall might wash out soiltransmitted helminth eggs from the soil $[7,34,35]$. The negative effects of precipitation seasonality in $T$. trichiura and hookworm models, precipitation in warmest quarter in A. lumbricoides model, as well as of soil moisture in hookworm model point in that direction. We also note that high isothermality, low range of temperature during the day and low precipitation seasonality are typical characteristics of northern equatorial and tropical humid regions of Brazil, suggesting that those climatic areas are suitable for transmission. The optimal soil acidity for
A. lumbricoides and T. trichiura transmission ranges between $\mathrm{pH}$ values of 5.35 and 5.65, however, hookworm prefers somewhat less acid conditions.

Our analysis also highlights the intimate connection of soil-transmitted helminth infection with poverty. Indeed, high percentage of poor households was an important risk factor for both T. trichiura and A. lumbricoides infections, after accounting for HDI. Poor households generally show lower frequencies of access and use of clean water and improved sanitation and thus are at higher odds of soil-transmitted helminth infection [36,37]. Another interesting aspect is the positive effect of HII and low percentage of rural households associated with T. trichiura risk, confirming previous findings $[5,7]$. These observations suggest that $T$. trichiura infection might be more prevalent in urban compared to rural settings [5].

Most of the soil-transmitted helminth data (97.5\%) stem from the Brazilian schistosomiasis control programme, which took advantage of the Kato-Katz technique, which allows concurrent diagnosis of soil-transmitted helminths, while screening for Schistosoma mansoni eggs in faecal thick smears. Brazil launched its national schistosomiasis control programme in 1975 with the aim of reducing schistosomiasis-related morbidity. Regarding soil-transmitted helminthiasis, the $\mathrm{MoH}$ re-started a 


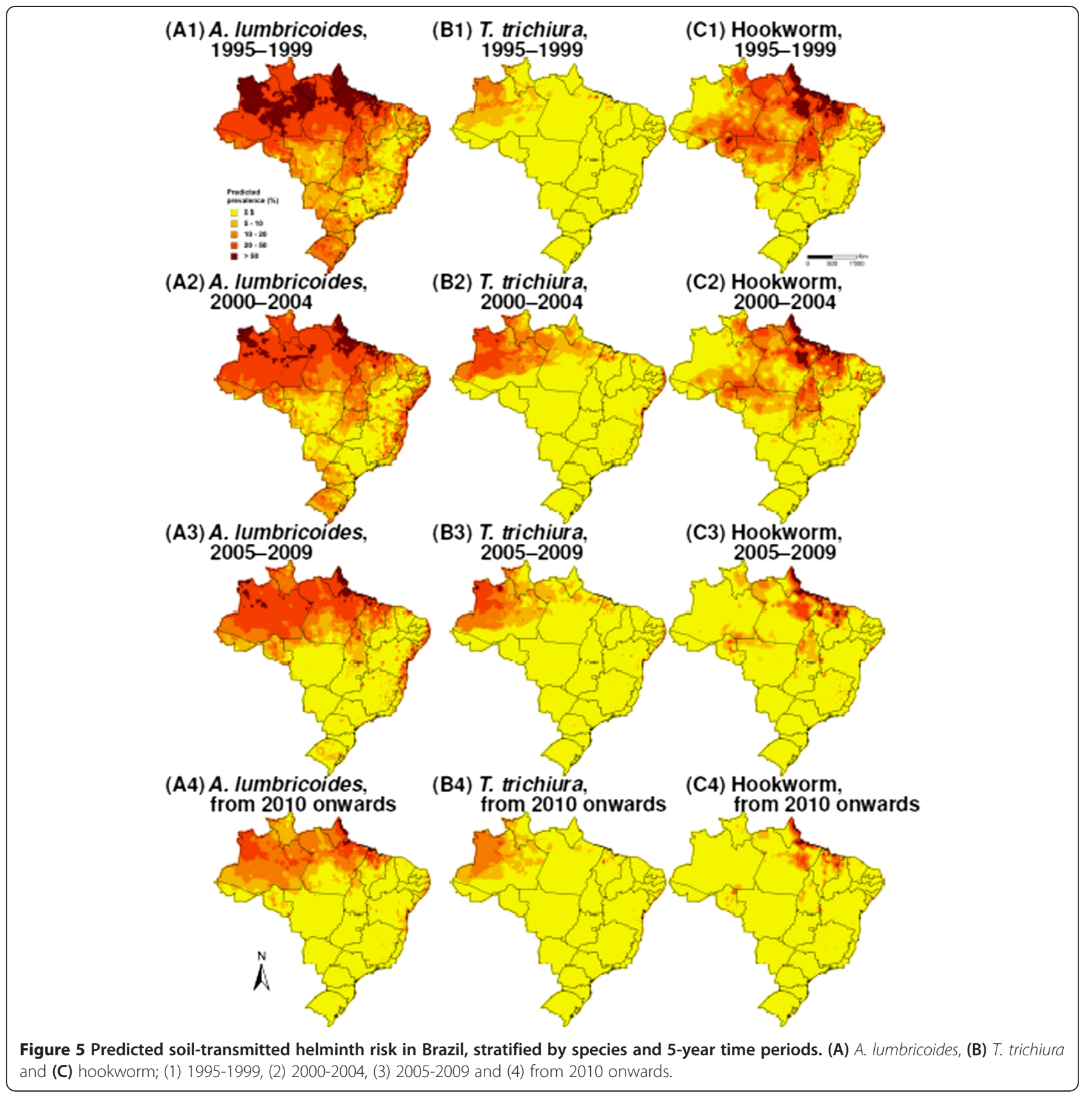

mass deworming campaign for school-aged children in 2013, prioritising areas characterised by a low HDI. This campaign will now be extended to the whole of Brazil. Data generated by this programme will facilitate the study of the evolution of the risks and evaluation of the impact of interventions. It will be important to specifically inform about the situation regarding the population targeted by the interventions (e.g. school-aged children or entire communities). Data that we analysed in the current study were mainly collected within the whole population (only 1\% referred to children exclusively). Thus, we might underestimate the risk among children, as it is known that they are usually at a higher risk of soil-transmitted helminth infections, particularly A. lumbricoides and T. trichiura [38]. Importantly though, despite low HDI showing a positive association with the three soil-transmitted helminth infection risks in bivariate associations, our spatio-temporal analysis indicates that helminthiasis risk was driven by complex environmental/socioeconomic interactions. Hence, we believe that our estimates provide useful information for a refined target of interventions. 
Table 6 Predicted population-adjusted risk of A. Iumbricoides, T. trichiura, hookworm and overall soil-transmitted helminth infection in Brazil, stratified by survey period

\begin{tabular}{lcccc}
\hline Survey period & $\begin{array}{c}\text { A. lumbricoides } \\
\text { infection risk (\%) }\end{array}$ & $\begin{array}{c}\text { T. trichiura } \\
\text { infection risk (\%) }\end{array}$ & $\begin{array}{c}\text { Hookworm } \\
\text { infection risk (\%) }\end{array}$ & $\begin{array}{c}\text { Soil-transmitted helminth } \\
\text { infection risk (\%) }\end{array}$ \\
\hline $1995-1999$ & $15.6(13.6 ; 18.0)$ & $1.8(1.4 ; 2.3)$ & $7.6(6.6 ; 9.0)$ & $20.9(19.0 ; 23.2)$ \\
$2000-2004$ & $11.4(10.0 ; 13.0)$ & $4.5(3.6 ; 5.7)$ & $5.7(4.9 ; 6.9)$ & $17.9(16.5 ; 19.7)$ \\
$2005-2009$ & $7.9(6.8 ; 9.1)$ & $2.5(2.1 ; 3.2)$ & $2.8(2.4 ; 3.4)$ & $11.5(10.4 ; 12.6)$ \\
From 2010 onwards & $3.6(3.0 ; 4.3)$ & $1.4(1.1 ; 1.7)$ & $1.7(1.4 ; 2.3)$ & $6.0(5.4 ; 6.9)$ \\
\hline
\end{tabular}

Population-adjusted risks are given with their $95 \%$ Bayesian credible interval (BCl).

Risk is adjusted on population of 2010 for survey periods from 2005 onwards and on population of 2000 for survey periods prior to 2005.

From a modelling point of view, it is important to highlight that SPDE methodology and INLA enabled us to fit our spatio-temporal models at a reasonable computational cost (around 4 hours per model, including fitting and prediction). Implementing this type of model would have been difficult to achieve with MCMC, especially because of convergence problems and large number of locations for spatio-temporal process estimation. With regard to the risk of the three soil-transmitted helminth species, residual spatial correlation was moderate (around $30 \mathrm{~km}$ ) and temporal autocorrelation was weak. Hence, most of the spatial and temporal dynamics were captured by the covariates of our models.

\section{Conclusions}

The methodology employed in the current analysis enables fitting more complex models and provides a useful tool for joint analysis of space and time components for risk profiling. The analysis of the spatiotemporal aspect of the risk of soil-transmitted helminth infections deepens our understanding of the evolution of the risk across time and enables more accurate predictions of the infection risks. We hope that our estimates will provide useful benchmark information for the soil-transmitted helminthiasis control programme in Brazil to prioritise interventions and enhance spatial targeting.

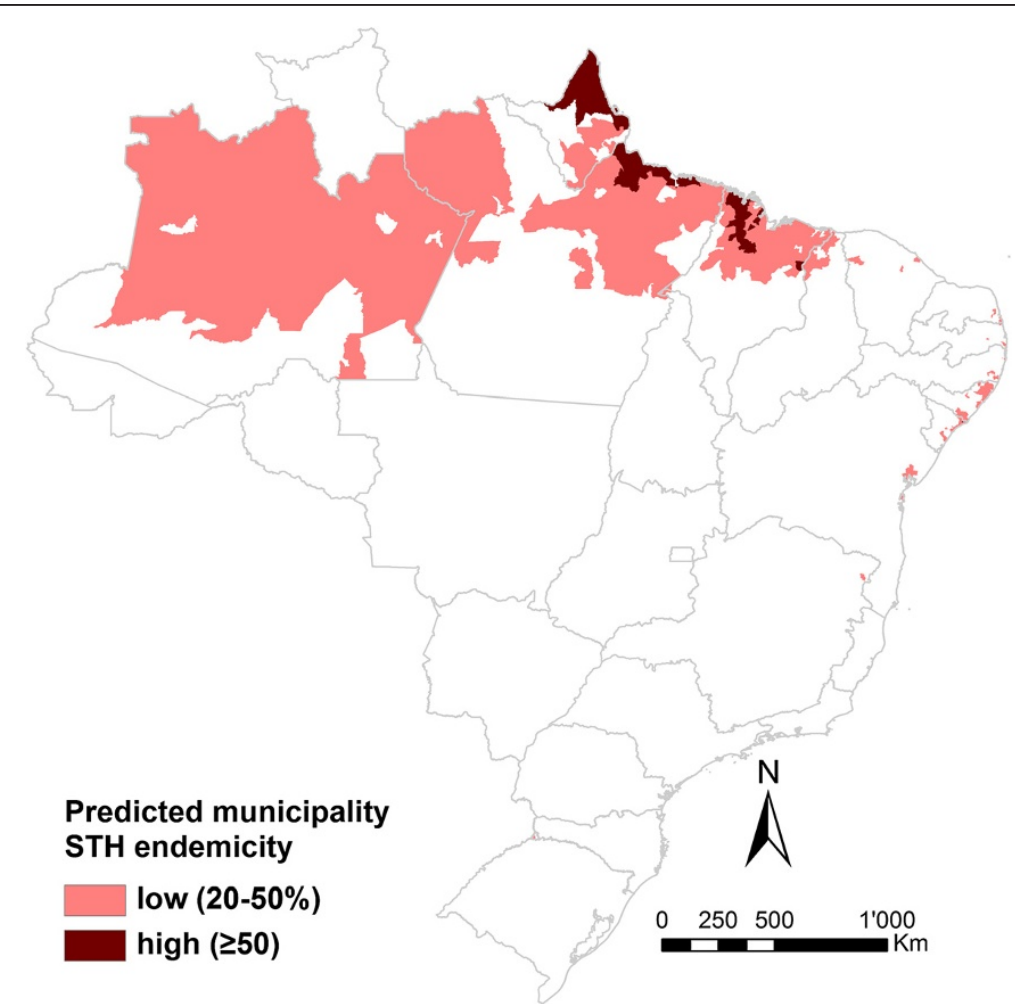

Figure 6 Estimated soil-transmitted helminthiasis (STH) endemicity of the Brazilian municipalities for intervention planning according to WHO guidelines pertaining to preventive chemotherapy. 


\section{Additional files}

\section{Additional file 1: Geostatistical variable selection and Bayesian} spatio-temporal model formulations.

\section{Abbreviations}

BCl: Bayesian credible interval; GF: Gaussian field; GMRF: Gaussian Markov random field; GNTD: Global Neglected Tropical Diseases (database); HDI: Human development index; HIl: Human influence index; HPD: Highest posterior density; INLA: Integrated nested Laplace approximation; MCMC: Markov chain Monte Carlo; ME: Mean error; $\mathrm{MoH}$ : Ministry of Health; peNMIG: Parameter expanded normal mixture of inverse-gamma; SPDE: Stochastic partial differential equation; WHO: World Health Organization.

\section{Competing interests}

The authors declare that they have no competing interests.

\section{Authors' contribution}

FC, JU and PV conceived and designed the study. FC, LHG and RGCS contributed to the systematic literature search and data extraction. RGCS and MEB participated in the environmental and socioeconomic data collection and helped interpreting their meaning. FC and PV analysed and interpreted the data. FC wrote the first draft of the manuscript. JU and PV assisted with the writing and revision of the manuscript. All authors read and approved the final version of the manuscript

\section{Acknowledgements}

The authors are grateful for financial support from the Swiss Brazilian Joint Research Programme (BSJRP 011008).

\section{Author details}

${ }^{1}$ Department of Epidemiology and Public Health, Swiss Tropical and Public Health Institute, P.O. Box, CH-4002 Basel, Switzerland. ${ }^{2}$ University of Basel, P.O. Box, CH-4003 Basel, Switzerland. ${ }^{3}$ Immunology Service, Hospital Edgard Santos, Federal University of Bahia, Salvador 40110-160, Bahia, Brazil. ${ }^{4}$ Coordenação Geral de Hanseníase e Doenças em Eliminação, Secretaria de Vigilância em Saúde, Brasília 70304-000, Distrito Federal, Brazil. ${ }^{5}$ Preventive Medicine Department, Federal University of Bahia, Salvador 40110-060, Bahia, Brazil.

Received: 30 April 2014 Accepted: 13 September 2014

Published: 18 September 2014

\section{References}

1. Bethony J, Brooker S, Albonico M, Geiger SM, Loukas A, Diemert D, Hotez PJ: Soil-transmitted helminth infections: ascariasis, trichuriasis, and hookworm. Lancet 2006, 367:1521-1532.

2. Hall A, Hewitt G, Tuffrey $V$, de Silva N: A review and meta-analysis of the impact of intestinal worms on child growth and nutrition. Mat Child Nutr 2008, 4:118-236.

3. Hotez PJ, Brindley PJ, Bethony JM, King CH, Pearce EJ, Jacobson J: Helminth infections: the great neglected tropical diseases. J Clin Invest 2008, 118:1311-1321.

4. WHO: Eliminating Soil-Transmitted Helminthiases as a Public Health Problem in Children. Progress Report 2001-2010 and Strategic Plan 2011-2020. Geneva: World Health Organization; 2012.

5. Pullan RL, Brooker SJ: The global limits and population at risk of soiltransmitted helminth infections in 2010. Parasit Vectors 2012, 5:81.

6. Pullan RL, Smith JL, Jasrasaria R, Brooker SJ: Global numbers of infection and disease burden of soil transmitted helminth infections in 2010. Parasit Vectors 2014, 7:37.

7. Chammartin F, Scholte RGC, Guimarães LH, Tanner M, Utzinger J, Vounatsou P: Soil-transmitted helminth infection in South America: a systematic review and geostatistical meta-analysis. Lancet Infect Dis 2013, 13:507-518.

8. Scholte RGC, Schur N, Bavia ME, Carvalho EM, Chammartin F, Utzinger J, Vounatsou P: Spatial analysis and risk mapping of soil-transmitted helminth infections in Brazil, using Bayesian geostatistical models. Geospat Health 2013, 8:97-110.
9. WHO: Soil-transmitted helminthiases: estimates of the number of children needing preventive chemotherapy and number treated, 2009. Wkly Epidemiol Rec 2011, 86:257-268.

10. Gabrielli AF, Montresor A, Nicholls RS, Ault SK: Progress towards the control and elimination of neglected tropical diseases in Brazil. J Pediatr (Rio J) 2013, 89:215-216

11. Diggle PJ, Tawn JA, Moyeed RA: Model-based geostatistics. J R Stat Soc Ser C Appl Stat 1998, 47:299-326.

12. Raso G, Vounatsou P, Gosoniu L, Tanner M, N'Goran EK, Utzinger J: Risk factors and spatial patterns of hookworm infection among schoolchildren in a rural area of western Côte d'Ivoire. Int J Parasitol 2006, 36:201-210.

13. Clements ACA, Deville MA, Ndayishimiye O, Brooker S, Fenwick A: Spatial co-distribution of neglected tropical diseases in the East African Great Lakes region: revisiting the justification for integrated control. Trop Med Int Health 2010, 15:198-207.

14. Lai YS, Zhou XN, Utzinger J, Vounatsou P: Bayesian geostatistical modelling of soil-transmitted helminth survey data in the People's Republic of China. Parasit Vectors 2013, 6:359.

15. Banerjee $S$, Gelfand $A E$, Finley $A O$, Sang $H$ : Gaussian predictive process models for large spatial data sets. J R Stat Soc Ser B Stat Methodol 2008, 70:825-848.

16. Chammartin F, Scholte RGC, Malone JB, Bavia ME, Nieto P, Utzinger J, Vounatsou P: Modelling the geographical distribution of soil-transmitted helminth infections in Bolivia. Parasit Vectors 2013, 6:152.

17. Knorr-Held L: Bayesian modelling of inseparable space-time variation in disease risk. Stat Med 2000, 19:2555-2567.

18. Rue H, Martino S, Chopin N: Approximate Bayesian inference for latent Gaussian models by using integrated Laplace approximations. J R Stat Soc Ser B Stat Methodol 2009, 71:319-392.

19. Lindgren F, Rue H, Lindström J: An explicit link between Gaussian fields and Gaussian Markov random fields: the stochastic partial differential equation approach. J R Stat Soc Ser B Stat Methodol 2011, 73:423-498.

20. Karagiannis-Voules DA, Scholte RGC, Guimarães LH, Utzinger J, Vounatsou P: Bayesian geostatistical modeling of leishmaniasis incidence in Brazil. PLoS Negl Trop Dis 2013, 7:e2213.

21. Hürlimann E, Schur N, Boutsika K, Stensgaard AS, Laserna de Himpsl M, Ziegelbauer K, Laizer N, Camenzind L, Di Pasquale A, Ekpo UF, Simoonga C, Mushinge G, Saarnak CFL, Utzinger J, Kristensen TK, Vounatsou P: Toward an open-access global database for mapping, control, and surveillance of neglected tropical diseases. PLoS Negl Trop Dis 2011, 5:e1404.

22. Saarnak CFL, Utzinger J, Kristensen TK: Collection, verification, sharing and dissemination of data: the CONTRAST experience. Acta Trop 2013, 128:407-411.

23. George El, McCulloch RE: Variable selection via Gibbs sampling. J Am Stat Assoc 1993, 88:881-889.

24. Scheipl F, Fahrmeir L, Kneib T: Spike-and-slab priors for function selection in structured additive regression model. J Am Stat Assoc 2012, 107:1518-1532.

25. Cameletti M, Lindgren F, Simpson D, Rue H: Spatio-temporal modeling of particulate matter concentration through the SPDE approach. Adv Stat Anal 2013, 97:109-131.

26. de Silva NR, Hall A: Using the prevalence of individual species of intestinal nematode worms to estimate the combined prevalence of any species. PLoS Negl Trop Dis 2010, 4:e655.

27. WHO: Preventive Chemotherapy in Human Helminthiasis: Coordinated Use of Anthelminthic Drugs in Control Interventions: A Manual for Health Professionals and Programme Managers. Geneva: World Health Organization; 2006.

28. Keiser J, Utzinger J: Efficacy of current drugs against soil-transmitted helminth infections: systematic review and meta-analysis. JAMA 2008, 299:1937-1948.

29. Keiser J, Utzinger J: The drugs we have and the drugs we need against major helminth infections. Adv Parasitol 2010, 73:197-230.

30. Oliveira MAG: Sources of Brazil's counter-hegemony. Rev Bras Polit Int 2010, 53:125-141.

31. Spindler $L A$ : The relation of moisture to the distribution of human Trichuris and Ascaris. Am J Epidemiol 1929, 10:476-496.

32. Brooker S, Clements ACA, Bundy DAP: Global epidemiology, ecology and control of soil-transmitted helminth infections. Adv Parasitol 2006, 62:221-261. 
33. Brooker S, Kabatereine NB, Tukahebwa EM, Kazibwe F: Spatial analysis of the distribution of intestinal nematode infections in Uganda. Epidemiol Infect 2004, 132:1065-1071.

34. Brown HW: Studies on the rate of development and viability of the eggs of Ascaris lumbricoides and Trichuris trichiura under field conditions. J Parasitol 1927, 14:1-15.

35. Gunawardena GS, Karunaweera ND, Ismail MM: Wet-days: are they better indicators of Ascaris infection levels? J Helminthol 2004, 78:305-310.

36. Ziegelbauer K, Speich B, Mäusezahl D, Bos R, Keiser J, Utzinger J: Effect of sanitation on soil-transmitted helminth infection: systematic review and meta-analysis. PLoS Med 2012, 9:e1001162.

37. Strunz EC, Addiss DG, Stocks M, Ogden S, Utzinger J, Freeman MC: Water, sanitation, hygiene, and soil-transmitted helminth infection: a systematic review and meta-analysis. PLoS Med 2014, 11:e1001620.

38. Anderson RM, May RM: Helminth infections of humans: mathematical models, population dynamics, and control. Adv Parasitol 1985, 24:1-101.

doi:10.1186/1756-3305-7-440

Cite this article as: Chammartin et al.: Spatio-temporal distribution of soil-transmitted helminth infections in Brazil. Parasites \& Vectors 2014 7:440.

\section{Submit your next manuscript to BioMed Central and take full advantage of:}

- Convenient online submission

- Thorough peer review

- No space constraints or color figure charges

- Immediate publication on acceptance

- Inclusion in PubMed, CAS, Scopus and Google Scholar

- Research which is freely available for redistribution 